\title{
Airport Management-Value of Customer Display Systems
}

\author{
Adam Marks', Maytha AL-Ali1 ${ }^{1}$, Kees Rietsema ${ }^{2}$ \\ ${ }^{1}$ Zayed University, Dubai, UAE \\ ${ }^{2}$ College of Business, Embry-Riddle Aeronautical University, Daytona Beach, USA \\ Email: marksa@eduval.net, alali@zu.ac.ae, rietsd37@erau.edu
}

Received 25 July 2015; accepted 20 September 2015; published 23 September 2015

Copyright ( 2015 by authors and Scientific Research Publishing Inc.

This work is licensed under the Creative Commons Attribution International License (CC BY).

http://creativecommons.org/licenses/by/4.0/

(c) () Open Access

\begin{abstract}
By providing real-time updates of essential information, airports not only display and disseminate information but also help control the flow of traffic. In order to maximize available space, particularly in high traffic areas, Airport Display Information Systems should be integrated into the overall design of the airport and their positioning should be carefully planned to deliver optimal results. Airport Display Information Systems can help airports maximize space, increase customer satisfaction, and generate new revenue opportunities. The technology is designed not only to comply with environmental regulations, but also to help airports keep budgets in check. This paper discusses airport display systems, their connections and interoperability with other systems and who the key airport users of these airport display systems are.
\end{abstract}

\section{Keywords}

\section{Airport, Aviation, Management Information Systems, Customer Display}

\section{Introduction}

The purpose of this paper is to contribute to the body of knowledge by providing descriptions of airport customer display information systems, their interoperability with other systems, and the key uses and users of each system. There are many types of customer display information systems and they can be organized or classified in a number of different ways [1] [2]. The system classification schema presented in this paper is neither all-inclusive nor exclusive; however, a number of leading aviation practitioners, business professionals, and educators in the industry are instrumental in both proposing and validating the schema. The study uses interviews, documentations, and observations as the primary sources of data. This paper is concerned with Customer Display Information Systems. Customer Display Information Systems provide easily accessible and detailed real-time infor- 
mation on flight schedules and airport services. Displaying information when and where it is needed is essential to customer satisfaction, which is reflected in the annual rankings of quality of goods, services, comfort, and convenience provided by airports.

By placing high in the national ranking, the airport's customer base, and thus profitability, may be increased. Customer Display Information Systems covered in this chapter are: Multi User Flight Information Display System (MUFIDS), Baggage Information Display System (BIDS), Airline Check-In-Desk Dynamic Display System (CDDDS), Way Finding and Dynamic Signage, System, and Airport Advertising System [1].

\section{Methodology}

This study uses qualitative methods to elicit data related to the classification and use of Landside Management Information Systems. The study collected data using observations, interviews, and document analysis. Twentyone extensive interviews were conducted with senior airport officials and IT directors from four US international airports and aviation faculty in Embry-Riddle Aeronautical University. A systematic search for the entire data corpus was conducted and data categories were created. Constant comparative method of data analysis was used (Merriam, 2001). The constant comparative method is a technique often used in the grounded theory tradition of qualitative research. It involves systematic search and arrangement of field notes and other data accumulated into categories in order to increase the understanding of the situation. In reviewing the field notes, the researchers generated and tested assertions by looking for key linkages and conducting member checks.

\section{Multi-Uses Flight Information Display System (MUFIDS)}

The Multi User Flight Information Display System (MUFIDS) encompasses the combination of monitors, networks, and software applications that provide the flight status on aircraft scheduled to land and depart an airport. The MUFIDS provides information-sharing, communication, and at some airports, marketing to customers. At most airports, the MUFIDS displays airline names, flight numbers, departure and arrival cities, estimated times of arrivals and departures, and departure and arrival gates. It also displays real-time flight status reported by direct data feeds from the FAA's information systems and airline information systems. The MUFIDS helps inform air travelers and airport visitors alike. For example, departing passengers can bypass customer service lines when the only information or check-in service they require is gate information and departure time. Individuals picking up arriving passengers can find out where to go without having to ask an airline customer service representative.

Passengers and visitors require up-to-date flight information from the moment they enter an airport's property perimeter and throughout their time in the airport facility. Therefore, for added convenience, MUFIDS monitors are installed in multiple locations throughout the airport, although the precise locations will differ between airports according to the strategies employed by the airport as part of its particular customer satisfaction program. The following is a list of the most common locations: aircraft ramp areas, airport baggage sorting areas, airport parking garages, airport rental car centers, airport train station lobbies, arrival-level baggage claim areas, commercial ground transportation areas (taxi and shuttle bus areas), common court areas for eateries and retail shops, security checkpoint entries, and ticketing lobbies and counters throughout the terminal.

Also for customer convenience, the MUFIDS orientation and format are standardized. To allow for the greatest use of the display area, large monitors are mounted in a vertical orientation so each category is a column. Plus, flight information is always listed alphabetically by arrival city and departure city. Use of the MUFIDS improves operational efficiency at an airport by providing real-time flight information generated directly by the FAA and the airlines. In the past, airports hired customer service employees to assist passengers with directions and flight information. Improvements and increased functionality in MUFIDS technology has allowed airports to reduce some of their customer service positions for answering flight-information questions.

Furthermore, the cost for maintaining the system is far less than the cost to employ customer service employees to provide the same information in all of the areas it is needed. Consequently, the MUFIDS meets the information needs of more customers and does so faster, cheaper, and more effectively compared to the traditional alternatives. Nonetheless, airport operators are continuously looking to expand and create revenue-generating opportunities. The modern MUFIDS can display advertising along with flight information. The display screen can be split into sections with the flight information on the main portion of the screen and advertising (banners, scrolling tickers, and pictures) shown on the other part of the screen. Public announcements are yet 
another use for the MUFIDS. Airport operators have discovered reducing the number of announcements over the airport PA system helps ensure security and safety audio broadcasts are heard and not ignored. The MUFIDS can be used to visually broadcast information such as gate changes for flights, pages to passengers, and airport security status and other messages. This feature is a significant benefit in high noise areas such as curbside check-in areas and security checkpoints. Continued advances in MUFIDS technology by manufacturers (e.g. Daktronics, Matrox Graphics, Industrial Television Services, Magenta Research, INFAX, INC, Com-Net, Parsons, MiTek Corporation, Net Display Systems, and SPEA Systems) have enabled airport operators to provide expanded and more efficient information services.

The management information connections include a network of computer hardware, software, and databases. Depending on the airport configuration, the MUFIDS application can receive data from more than one source, including the FAA, airlines, and product vendors. The MUFIDS may be maintained by the airport operator through a common-use arrangement or by one or more airlines under an exclusive-use arrangement. In the common-use arrangement, the airport purchases a software application designed to manage the MUFIDS. The application is configurable so the MUFIDS monitors can display information in the format required by the airport operator. Regardless of who maintains the system, proper data management and configuration of the application is essential to ensure information displayed on the MUFIDS is current. The data entered into the MUFIDS consists of information about airport equipment, including each connected display monitor and the routing equipment that sends the proper information to the appropriate monitors. Depending on the MUFIDS, an application such as WinFIDS can manage the information displayed by presenting certain information at preconfigured locations and times. For example, the system administrator can program flight data imported into the MUFIDS to display at certain locations at specific time intervals and remain on the monitors for specific lengths of times. Data can be entered automatically into the application from the airport's interconnected Gate Management System or it can be entered manually by the system administrator or anyone with the appropriate access rights or system permissions. If the MUFIDS application accepts data feeds automatically from the Gate Management System, system monitors will display up-to-date boarding gate assignments and arrivals of unscheduled flights. In an exclusive-use tenant agreement, an airline provides information directly into the MUFIDS from its flight scheduling system such as SABRE to populate the appropriate MUFIDS screens with its flight information. Each airline is responsible for ensuring their data remain up-to-date, so their customers will be viewing the correct information on the MUFIDS displays. This is made possible either through a direct feed from the airline's flight-scheduling system via wireless Internet connections or through hardwired connections.

An airline may use commercial or customized software to enter data for its routine flights and the airport equipment needed for processing scheduled flights (e.g. boarding gates, baggage claim carousels, and MUFIDS monitors). Data may also be relayed through connections with a global vendor of the MUFIDS software and services. In this situation, the airline provides the product vendor with its data feed and the vendor relays the information to the MUFIDS at each airport that the airline flies into and out of around the world. With all of these arrangements, data feeds from the airlines populate MUFIDS databases with real-time flight information.

The MUFIDS software is a behind-the-scene application requiring limited involvement by airport employees once the system is installed and configured properly. The software provides features that allow system operators to modify the appearance of the MUFIDS display, including changing the color, font, and layout of the information. The software may also have the capability to add custom advertising and visual paging messages. In a common-use configuration, the MUFIDS application interfaces with the different airlines' flight-scheduling systems and Gate Management Systems to display their flight schedules along with the scheduled departure and arrival gates. The basic hardware components are similar for most MUFIDS installations. The front end consists of the monitors that are visible to airport customers. These may be LED (Light Emitting Diode), LCD (Liquid Crystal Display), CRT (Cathode Ray Tube), or plasma displays. The sizes of the monitors typically range from 9 to 46 inches, but may be larger, even as large as 40 to 50 inches. The intended use, display resolution, colors, planned content, and installation locations are all considerations for selecting and deploying MUFIDS monitors. Resolutions of 1280 pixels by 768 pixels are common for large LCDs while plasma displays have resolutions between 853 pixels by 480 pixels or 1024 pixels by 768 pixels. The best brightness and contrast ratio for each monitor depends on the specific location of its installation. Layers of servers are connected to the display monitors. In some installations, mini severs are located near the display but out of the customers' view. Video cards are installed in the mini servers connected to multiple monitors (usually four per server). These servers are connected throughout the airport's LANS and are integrated with the back-end servers that process the data sent to the dis- 
plays. An airport MUFIDS can run on an existing airport LANS or on a separate network if the system installation and maintenance are outsourced. The basic requirement for the MUFIDS operation includes an Internet connection that allows the software application to request flight information from airline databases or the vendor database and FAA databases. The network configuration is dependent upon the MUFIDS application, whether or not the vendor is maintaining the system, and which airlines are using the system. Airports with older MUFIDS installations may also have serial connections to the network providing data feeds through RS-232 connectors.

Data for the MUFIDS is stored on Microsoft ${ }^{\circledR}$ SQL or Oracle ${ }^{\circledR}$ application servers. Stored data include the airport equipment used to both configure and manage the software that displays the flight-scheduling data on the MUFIDS monitors. Flight-scheduling data include information about projected and actual flights for the airport and the individual airlines that have a common-use or exclusive-use agreement with the airport. Flight data imported into the MUFIDS usually include the flight number, estimated arrival, actual arrival, scheduled gate, estimated departure, actual departure, arrival and departure city, and current flight status (on time, delayed, canceled, etc.). The database allows information from each airline to be imported into airline specific object tables. The MUFIDS application time stamps and tracks each data entry to the MUFIDS database; thus, allowing system operators to trace errors back to their source. In most airports, the IT employees maintain the network equipment and, in some cases, serve as system administrators to assign access rights and make configuration changes to the MUFIDS application. However, some airports use third-party contractors to maintain their system and make updates and changes, although the IT department still has administrator rights with full access for system audits and to provide network support.

\section{Baggage Information Display Systems (BIDS)}

The Baggage Information Display System (BIDS), an interoperable system with the MUFIDS, encompasses the baggage-belt displays that inform deplaned passengers which baggage carousel to go to for retrieving their checked luggage. The BIDS includes monitors for displaying baggage retrieval information for passengers and airport customers. These monitors are typically located in the airport's baggage claim lobbies, near the baggage carousels.

The BIDS monitors interface with the same software application that manages the MUFIDS, so they receive the same data from the airport's Gate Management System. The data displayed includes the aircraft flight number, departing city, airport, and gate, arriving city, airport and gate, and the baggage carousels distributing checked luggage. As with the MUFIDS, the BIDS can also display advertising, announcements, airport security messages, and pages to customers. Assignments of the baggage carousels depend on each airline's agreement with the airport. At large hub airports, the airlines have priority agreements granting them exclusive use of assigned baggage carousels.

Those carousels are in the database for flight scheduling, so are automatically assigned with the flight by the airline's flight scheduling program. Non-hub airlines that require baggage carousels for distributing their passengers' checked luggage are scheduled by the airport's Gate Management System.

When an aircraft arrives at its destination airport, baggage handlers offload the checked luggage onto baggage carts. From there the luggage is transported to the baggage make-up areas where it is loaded onto the ramp-side baggage carousels that transport it to the baggage carousels identified on the BIDS monitors. Airline employees are able to make last minute changes to baggage carousel assignments through a BIDS keyboard at each workstation. Use of the BIDS improves operational efficiency and reduces costs for airports by providing real-time baggage information to passengers and visitors. As with the MUFIDS, the system also provides revenue opportunities for airports by allowing companies to advertise to passengers and visitors. The BIDS is capable of integrating with various airport systems, so can be purposed as an emergency alerting system when needed. The features and functionality provided by the BIDS allows an airport to reach customers in unconventional and yet effective ways.

The BIDS management information connections require computer hardware, networks, software, and databases. In most airports, the same software used to manage the MUFIDS is also used to manage the BIDS. The software allows the primary users to change the display layout and update the data to reflect up-to-the-minute changes in flight schedules and baggage carousel assignments. The hardware includes the same equipment as that needed for the MUFIDS, including monitors, communications equipment, interface computers, and database servers. Consequently, the BIDS does not require a separate database because much of the information is 
the same data stored for the MUFIDS, including the configurations and locations for the display monitors. The BIDS can be setup to run on the airport's LAN, on an airline's LAN, or on a separate network installed by the product vendor or contractor hired to maintain and operate the system. Therefore, the airport's IT personnel provide the same network administration and maintenance as they do for the MUFIDS, unless these services are outsourced to a commercial vendor or contractor.

\section{Check-in-Desk Dynamic Display System (CDDDS)}

The Check-In-Desk Dynamic Display System (CDDDS) encompasses the check-in information displays located behind airline ticketing counters that can be programed to show airline branding, messages such as passenger queuing for the different types of ticketing lines, or airport advertising. Technology has advanced to the point where air travelers are able to purchase tickets, check luggage, and obtain the status of their flight before ever going to the airport.

There are, however, situations where they need to visit an airline check-in ticket counter for assistance. Dynamic displays located behind and above these check-in counters can be programed to show any type of flight information in a digital format. Airlines display their name or trademarked brand on their CDDDS displays to direct customers to their check-in counters. However, most check-in-desk dynamic displays belong to the airport; so an airline must be granted use of them in either a priority-use agreement or common-use agreement with the airport operator.

Airlines with priority-use agreements with the airport control all of the equipment at their ticketing desks, including the check-in displays. Information displayed on check-in screens comes directly from the airline ticketing check-in system or through the airport's Common-Use Passenger Processing System (CUPPS); formally referred to as Common Use Terminal Equipment (CUTE). An airline employee signs into an authorized workstation where a terminal-emulation service provides direct access to the airline's operating environment. The employee is able to see the same GUI that he or she would see if they were using a ticketing workstation owed by the airline. Once logged into the system, the check-in desk displays installed at the airline's ticketing-counters will show the information chosen by the airline. When used by the airlines, the check-in displays provide everyone in the vicinity of the airline's ticketing counters with the airline's arrivals and departures as well as information directed to types of ticket holders (e.g. first class, business class, economy class), and any other information programed by the operator. By not having to answer the common types of travel questions, airline-ticketing agents are able to process significantly more passengers; thus, ticket-counter operations have become more efficient. These check-in displays can also provide revenue-generating opportunities for the airport. The airport operator uses these displays for marketing airport services provided by its licensed vendors when airline user agreements are nonexclusive.

The management information connections include the airlines' systems, the airport's Gate Management System, and CUPPS. The CDDDS is comprised of a series of monitors networked to a computer system that transmits information to the airline check-in display monitors directly from the computer system managing the ticketing application. This configuration also relies on the airline's network for data transmission between devices. In contrast, check-in monitors connected to the Gate Management System access data programmed by the administrator or generated by the terminal-emulation program. Hardware equipment includes the monitors that display information at the check-in counters for passengers and also monitors and keyboards for airline employees to use to control the type of information displayed and how that information is configured on the screens. The information transmits over the airport's LANS when the CDDDS is nonexclusive or over an airline's LANS when the airline has an exclusive-use agreement with the airport. Network equipment includes routers, switches, controllers, servers, and computers to provide input to the displays. The check-in display screens do not require a database for storing information because information is pulled from the airline's ticketing system or the airport's Gate Management System. Furthermore, the information on most of the check-in-displays is static, so they do not require database connections. With the common-use approach, configuration and control of the check-in display system is conducted behind-the-scenes either by airport IT personnel or the vendor contracted to manage the system. With the exclusive-use approach, airline personnel manage the display system to convey their current flight schedules and other information dedicated for their passengers. In the common-use approach, the airport's IT department is responsible for configuring and maintaining the CDDDS to display airport information when the displays are not in use by the airlines. They also provide airlines with access rights to the sys- 
tem so their employees are able to establish connections to the terminal emulator whenever necessary. The IT service personnel also repair and replace hardware equipment to keep the system operational.

\section{Wayfinding and Dynamic Signage Systems}

The Wayfinding and Dynamic Signage System encompasses both static wayfinding signage and dynamic electronic signage that provides wayfinding, marketing, security, or emergency information to airport customers and employees while on airport property. Airport operators are constantly seeking new ways of improving customer service and convenience. One method that has proven quite effective is empowering airport visitors to self-select the information they need to make informed decisions; thus, avoiding relying on airport or airline employees for information.

Given the nature of tight flight schedules, this strategy helps make the airport experience less time consuming and stressful for passengers and visitors alike. Dynamic signs allow the airport operator to provide passengers, visitors, and airport employees with information about the airport. This information can range from the location of the nearest restrooms to locations of restaurants. Dynamic signs allow the airport operator to provide more information by eliminating the need for static displays like poster boards. Dynamic signs are interoperable with the MUFIDS, paging, and ticketing systems, so operators can program the signs to display any information and do so for varying time spans [3].

The most common questions from airport customers are how to get to the departure/arrival gates, convenience facilities, and ground transportation. In the past, they had no choice but to obtain this information from static signage or from customer-service employees stationed sporadically throughout airport terminals. This wayfinding process was often frustrating if not also confusing, especially for passengers not fluent in the native language. Dynamic signs have all but eliminated this problem by being able to display wayfinding information in any language in numerous convenient locations in the airport. Indeed, dynamic wayfinding signage can be located almost anywhere. They can be installed over airport roadways, inside parking structures, at arrival/departure curbsides, ticketing lobbies, baggage facilities, concourse gates, and aircraft ramps.

The management information connections include remote and mobile inputs from users authorized to change the data delivered to the displays. Software applications are available that allow the airport personnel to create signage specific to the airport by incorporating the airport's theme within the displays. The system can be integrated into the Gate Management System, MUFIDS, emergency notification systems, and paging systems. The system can also integrate with the airport's GIS and website. System hardware ranges from LED signs to electronic high-resolution monitors and high-powered video processing equipment, computer servers, and network equipment. The system can reside on the airport's LANS and connect to other systems via the established LANS or connect through a Web-interface application for management and system updates. Data for the system may reside on existing airport servers dedicated for the signage system or on servers from the company or companies contracted to manage the system. Stored data may range from specific information about the airport layout to specific locations and services for each tenant. Airlines and other tenants may also choose to purchase advertising space on the dynamic signs to provide information specific to their operations or business. Therefore, the data for most of the dynamic signage can be stored within existing airport databases applications or on database applications maintained by third-party vendors. Airport IT employees usually serve as primary operators of dynamic signage system. They may be responsible for programming and updating the signage or they may only serve as system administrators overseeing the work performed by contractors and third-party vendors. The IT personnel may also provide access rights to authorized operators, maintain the hardware and database, and perform software integration needed to operate the dynamic signage.

\section{Website Advertising Systems}

The Website Advertising System is an airport's commercial presence on the World Wide Web via the Internet that informs the public and stakeholders about the airport, including its location, flights, services, and much more. Airport websites must be dynamic and interactive to allow users to obtain information and perform many of the tasks once relegated to person-to-person interactions over the telephone or inside the airport. Visitors to an airport website are able to obtain real-time flight information, complete request forms, access dynamic maps of the airport, and even apply for an airport job or contract. General information about the airport is displayed on or linked from the landing page called the Homepage. Information usually includes airport history, contact in- 
formation, location, and customer services.

The homepage also incorporates embedded hyperlinks to other Web pages that display pictures, diagrams, and information on the airport facility, terminal buildings, runways, taxiways, parking areas, passenger check-in areas, baggage-claim areas, and passenger pickup areas. A common practice is to hyperlink images so that when a website visitor clicks on an image, the Web page jumps to a different Web page or pops up and plays a video. Maps are usually interactive. The user can click and pan the view or zoom in to see a detailed map or zoom out to see a large-scale map of the airport and its surroundings. Specific driving directions to the airport can be obtained by simply entering an address from which to navigate. There may be hyperlinks to information about the airport's senior leadership team as well as the airport master plan that describes planned construction and development projects for the next 10 years. There is usually a Web page for Frequently Asked Questions (FAQ) providing answers to the most commonly asked questions.

Airline information encompasses the active airlines that do business at the airport. This includes all regularly scheduled domestic and international passenger airlines, charter airline companies, cargo airlines, and general aviation airport facilities. Flight information comes directly from flight-tracking services such as Track-A-Flight or Flight View. The databases for the flight-tracking services have direct links to FAA feeds and provide access to the airlines so their operators can enter information about prospective flights. These services use XML formatted data feeds that provide flight schedules and real-time departure and arrival information, flight delay and cancellation information, real-time airport delay information, and airline and aircraft flight information. Consequently, website users receive current flight information from the airlines and up-to-the minute status on airline flights as updated by the FAA. The website provides information for other people such as prospective vendors and airport contractors interested in doing business with the airport. This section of the website often contains information on upcoming projects available for vendor and contractor bidding. Such users may be able to retrieve specifics on airport contracts, procurement processes, business diversity policies, rates and charges, cargo operating requirements, airline competition plans, and other employment opportunities. Users can request additional services and enroll in the selection process for projects and open positions with the airport operator. Airport parking is such an important topic to airport visitors and passengers that parking status information is being integrated into airport websites to inform passengers of the capacities of their parking decks and alert them when parking lots are closed. Some airports even display the number of available parking slots and locations based on the expected time of arrival at the airport input by the user. Interactive maps may be used to provide relevant information on retail establishments, restaurants, and other types of customer services. Food and beverage concessions within the terminal building and concourses are identified so visitors know exactly where to go for these types of amenities in the airport.

A dynamic website can increase efficiency, reduce cost, and generate revenue for the airport by incorporating electronic marketing tools and providing detailed information that entices users with vivid and relevant descriptions of airport services. Airport website developers ensure the design both attracts and interests visitors in discovering new services and amenities offered by the airport and they ensure the airport information is accurate and current to help guarantee repeat visits. A website with rich, dynamic, interactive content improves communications between the airport operator and its customers. Plus, directing customers to the solutions provided online lowers the number of telephone calls for information as well as the time spent on the telephone answering customer service questions. Besides the benefits to website visitors, an effective website improves airport efficiency by serving more customers and servicing them faster and without the need for human interaction, which results in monetary savings for the airport The management information connections require website development software such as ASP.NET for creating dynamic and interactive user experiences that range from dynamic searching, mouse-over hyperlinks, and integration of Adobe Flash and HTML5 for videos. Web developers typically integrate a host of other programs including Microsoft Silverlight ${ }^{\circledR}$ and Visual Studio ${ }^{\circledR}$ and Adobe Flash ${ }^{\circledR}$ and Dreamweaver ${ }^{\circledR}$ into their development processes. Many developers obtain programming scripts from companies such as Google ${ }^{\circledR}$, Yahoo ${ }^{\circledR}$, and YouTube ${ }^{\circledR}$ for integrating common Web apps and gadgets into the airport's website.

Examples include programming scripts for a Google ${ }^{\circledR}$ search engine or Yahoo ${ }^{\circledR}$ weather or time dashboards. The hardware required to host the website is dependent on who develops the website and how it is developed. When an airport IT department develops and hosts the website, a Web server is needed to store information in a database and network equipment is needed to establish an ISP connection to the airport's website. Examples of server operating platforms include Windows Server $2003 \AA$, Windows Server $2008 \AA$, Linux ${ }^{\circledR}$, UNIX ${ }^{\mathrm{TM}}$, and 
Mac OS®. On the back end of the website, multiple interconnected Web servers allow use of only one public IP address. Selection of the type of Web server depends on the applications and operation systems being used. Website hardware varies between one airport website to the next depending if the development is done in-house by the airport's IT department or it is outsourced. When the site is developed in-house, the airport's IT professionals are responsible for creating the content, designing the web pages, maintaining the website, and providing secure access to the site. This requires the purchase and installation of hardware, software, and networking equipment. A software platform such as Oracle ${ }^{\circledR}$ is needed to store information in object tables in a relational database. The database provides the capabilities for interactive maps and request forms, but also stores data on the airport's amenities, facilities, airlines, and tenants. Regardless if the development of the airport website is done in-house or by an outside consultant, the airport's IT professionals are responsible for creating the content, designing the web pages, maintaining the website, and providing secure site access.

\section{Conclusion}

Displaying information when and where it is needed is a key factor in the annual rankings of quality of goods, services, comfort, and convenience provided by airports. The airport experience plays a major role when customers choose between airports. Customer Display Information Systems are a major part of the customer's experience, as they provide guidance, ease, convenience, and stress free experience. The customer display information systems covered in this paper are: Multi User Flight Information Display System (MUFIDS), Baggage Information Display System (BIDS), Check-in-Desk Dynamic Display System (CDDDS), Wayfinding and Dynamic Signage, System, and Airport Advertising System [1]. Similar to other information systems, customer display information systems incorporate software, database, and network components. They generate both ondemand and regular reports to assist airport management improve operations and reduce cost at the same time. This paper provides a possible classification for airport customer display systems and describes some of their use, benefits, and limitations.

\section{References}

[1] Marks, A., Rietsema, K. and Hudson, G. (2013) Aviation Managmenet Information Systems. Embry-Riddle Press.

[2] Airport Cooperative Reseach Program (2008) Integrative Airport Information Systems-Report 13. Transporation Research Board.

[3] (2010) ACRP Synthesis 21 Airport Energy Efficiency and Cost Reduction. Transportation Research Board. 